\title{
Neuroprotective Effects of Vascular Endothelial Growth Factor Following Hypoxic Ischemic Brain Injury in Neonatal Rats
}

\author{
YANGZHENG FENG, PHILIP G. RHODES, AND ABHAY J. BHATT
}

Department of Pediatrics, University of Mississippi Medical Center, Jackson, Mississippi 39216

\begin{abstract}
Vascular Endothelial Growth Factor (VEGF) protects the brain against ischemic injury in adult animals. We evaluated whether VEGF has neuroprotective effects against hypoxic-ischemic (HI) brain injury in newborn rats. Seven-day-old rat pups had the right carotid artery permanently ligated followed by $140 \mathrm{~min}$ of hypoxia (8\% oxygen). VEGF $(5,10,20$, or $40 \mathrm{ng})$ or vehicle was administered intracerebroventricularly $5 \mathrm{~min}$ after reoxygenation following HI. Brain damage was evaluated by weight loss of the right hemisphere at $22 \mathrm{~d}$ after $\mathrm{HI}$ and by gross and microscopic morphology. Body weight, rectal temperature, and mortality were not significantly different in the VEGF and vehicle treated groups. VEGF treatment increased brain VEGF levels at $15 \mathrm{~min}$ after injection. VEGF (10 and $20 \mathrm{ng}$ ) significantly reduced brain weight loss $(p<$ 0.05 ) and gross brain injury $(p<0.05)$; however, treatment with 5 or $40 \mathrm{ng}$ did not. VEGF (10 ng) also decreased brain damage assessed by histologic scoring. VEGF increased phosphorylation of protein kinase $\mathrm{B}(\mathrm{Akt})$ and extracellular-signal regulated kinase $1 / 2$ (ERK1/2) in the cortex $(p<0.05)$. These results suggest that VEGF has neuroprotective effects in the neonatal rat $\mathrm{HI}$ model that may be related to activation of the Akt/ERK signaling pathway. (Pediatr Res 64: 370-374, 2008)
\end{abstract}

$\mathrm{N}$ eonatal hypoxic-ischemic (HI) cerebral injury, the most common known cause of cerebral palsy, is an evolving process that is initiated during the insult and extends into a recovery period, the "reperfusion phase," which is amenable to potential intervention(s) $(1,2)$. Novel strategies aimed at preventing ongoing injury are being clinically evaluated and offer an opportunity for neuroprotection (3). The neonatal rat model of HI has been well characterized (4) and used extensively to assess the efficacy of neuroprotective agents (5).

Vascular Endothelial Growth Factor A (VEGF), an angiogenic growth factor and survival factor for endothelial cells, also exhibits neurotrophic and neuroprotective effects. Exogenously applied VEGF enhances survival of mesencephalic neurons in organotypic explant cultures (6). VEGF also reduces hypoxic death of both immortalized hippocampal neuronal cells and cultured cerebral cortical neurons $(7,8)$. Recent data suggest that VEGF protects the brain against ischemic injury in adult animals (9-11), but limited information is available in newborn animals.

Evidence suggests that apoptosis contributes significantly to injury in the delayed phase after milder degrees of neonatal HI

Received January 22, 2008; accepted May 6, 2008.

Correspondence: Abhay J. Bhatt, M.D., Department of Pediatrics, University of Mississippi Medical Center, 2500 North State Street, Jackson, Mississippi 39216-4505; e-mail: abhatt@ped.umsmed.edu
(12). The serine-threonine kinase, protein kinase B (Akt) is one of the major downstream pathways of neurotrophin signaling and plays a critical role in controlling the balance between survival and apoptosis (13). Activation of Akt has been shown to promote neuronal survival after ischemia $(14,15)$. Extracellular Signal-Regulated Kinases (ERK 1/2 or ERKs) are known to be involved in cell proliferation, differentiation, and survival. Functional relevance of ERKs activation depends upon the type of cell and the nature of the stimulus (16). Evidence suggests they have a role in the induction of tolerance to ischemic injury in heart, adult brain, and neonatal brain (16). Previous studies have suggested that the molecular mechanisms of neuroprotective effects of VEGF involve activation of Akt and/ or ERKs $(7,17,18)$.

In the present study, we investigated whether VEGF treatment would reduce brain injury in the neonatal rat HI model and the potential mechanisms of neuroprotection.

\section{MATERIALS AND METHODS}

\begin{abstract}
Animal protocol. The protocol was approved by the University of Mississippi institutional committee on animal use. Rats were cared for in accordance with National Institutes of Health guidelines. The neonatal rat $\mathrm{HI}$ procedure was performed as described by Rice et al. (4) with minor modifications. Seven-day-old Sprague-Dawley rat pups of either sex, weighing between 12 and $16 \mathrm{~g}$ (Charles River Laboratories, Wilmington, MA) were anesthetized with $3 \%$ isoflurane. The right common carotid artery was exposed, isolated and permanently doubly ligated. After surgery, the rat pups were returned to their dams for $2-3 \mathrm{~h}$ recovery. Hypoxic exposure was achieved by placing the rat pups in 1.5-1 sealed jars immersed $5.5-\mathrm{cm}$ deep in a $37^{\circ} \mathrm{C}$ water bath and subjected to a warmed, humidified mixture of $8 \%$ oxygen/92\% nitrogen bubbled through $37^{\circ} \mathrm{C}$ water and delivered at $4 \mathrm{l} / \mathrm{min}$ for $140 \mathrm{~min}$. After this hypoxic exposure, the pups were returned to their dams and allowed to recover and grow for the following experiments. Pups were euthanized and brains were collected at different time points for brain VEGF levels, brain water content, Akt and ERKs assay and were then studied 3 and $22 \mathrm{~d}$ after HI for brain injury measurement.

Drug treatment. Pups from each litter were randomly assigned, marked and treated with VEGF (human recombinant VEGF $_{165}$, Sigma Chemical Co.-Aldrich Co., St. Louis, MO) or vehicle according to the method described by Cheng et al. (19) with minor modification. VEGF 5, 10, 20, or $40 \mathrm{ng}$ in 2 $\mu l$ phosphate-buffered saline (PBS) was administered intracerebroventricularly (i.c.v.) $5 \mathrm{~min}$ after reoxygenation following $\mathrm{HI}(n=19-33$ in each, see Table 1). These doses were chosen from a previous study (11). The corresponding vehicle-treated pups were given the same volume of PBS $(n=$ 19-29 in each, see Table 1). The location of each injection in relation to lambda was $2.0-\mathrm{mm}$ rostral, $1.5-\mathrm{mm}$ lateral, and $2.0-\mathrm{mm}$ deep to the skull surface. The sham-operated pups $(n=20)$ were treated similarly to the operated ones, except that the carotid artery was not ligated and they were not exposed to hypoxia.
\end{abstract}

Abbreviations: Akt, protein kinase B; ERK, extracellular-signal regulated kinase; HI, hypoxic-ischemic; i.c.v., intracerebroventricular 
Table 1. Gross damage score

\begin{tabular}{lccccc}
\hline \multicolumn{1}{c}{ Group } & $N$ & $\begin{array}{c}\text { Normal } \\
(\%)\end{array}$ & $\begin{array}{c}\text { Mild } \\
(\%)\end{array}$ & $\begin{array}{c}\text { Moderate } \\
(\%)\end{array}$ & $\begin{array}{c}\text { Severe } \\
(\%)\end{array}$ \\
\hline Vehicle & 19 & $5(26.3)$ & $7(36.8)$ & $7(36.8)$ & $0(0.0)$ \\
VEGF 5 ng & 19 & $8(42.1)$ & $4(21.1)$ & $5(26.3)$ & $2(10.5)$ \\
Vehicle & 26 & $7(26.9)$ & $6(23.1)$ & $7(26.9)$ & $6(23.1)$ \\
VEGF 10 ng & 25 & $15(60.0)^{*}$ & $6(24.0)$ & $4(16.0)$ & $0(0.0 *$ \\
Vehicle & 29 & $10(34.5)$ & $9(31.0)$ & $8(27.6)$ & $2(6.9)$ \\
VEGF 20 ng & 33 & $22(66.7)^{*}$ & $5(15.2)$ & $6(18.2)$ & $0(0.0)$ \\
Vehicle & 20 & $4(20.0)$ & $9(45.0)$ & $4(20.0)$ & $3(15.0)$ \\
VEGF 40 ng & 20 & $7(35.0)$ & $3(15.0)$ & $6(30.0)$ & $4(20.0)$ \\
\hline
\end{tabular}

$* p<0.05$ vs. vehicle.

Measurement of rectal temperature and body weight. To evaluate whether neuroprotection by VEGF was dependent on systemic hypothermia, rectal temperature was measured with a 36-gauge flexible thermocouple (Omega Engineering Inc., Stamford, CT). This was done in 12 pups from 1 litter (six from the vehicle group and six given $10 \mathrm{ng}$ of VEGF) before hypoxia; and at 0 and $0.25,0.5,1,1.5,2,2.5,3$, and $4 \mathrm{~h}$ after reoxygenation following $\mathrm{HI}$.

Body weight was measured in the rat pups (vehicle, $n=88$; VEGF $5 \mathrm{ng}$, $n=19,10 \mathrm{ng}, n=19,20 \mathrm{ng}, n=33$, or $40 \mathrm{ng}, n=20$ ) at $0,1,4,7,11,14$, and $22 \mathrm{~d}$ after injury.

Measurement of brain water content. To examine whether VEGFs beneficial effect might be offset by increased cerebral edema, brain water content was measured. Using the above neonatal $\mathrm{HI}$ procedure, the rat pups were treated with vehicle or VEGF. At $24 \mathrm{~h}$ after HI, the pups were decapitated, the brains rapidly removed and the hemispheres were divided. The samples were weighed and then dried at $95^{\circ} \mathrm{C}$ for $48 \mathrm{~h}$ to obtain the dry weight. The brain water content was calculated as [(wet weight - dry weight)/wet weight $\times 100]$.

Measurement of brain levels of VEGF protein. Using the above neonatal $\mathrm{HI}$ procedure, the rat pups were treated with vehicle or $10 \mathrm{ng}$ of VEGF. At 15, $45 \mathrm{~min}, 6,24 \mathrm{~h}, 2$ and $3 \mathrm{~d}(n=5-8$ in each group) after administration, the pups were decapitated, the brains removed, the cortexes (right hemisphere) were dissected and frozen at $-80^{\circ} \mathrm{C}$. VEGF protein levels were determined using the Quantikine, mouse VEGF Immunoassay kit ( $\mathrm{R} \&$ D Systems, Minneapolis, MN) according to the manufacturer's instructions. This assay employs the quantitative sandwich enzyme immunoassay technique and recognizes all major isoforms of VEGF. The levels of VEGF protein in the brain were normalized and expressed as picograms per milligram of total brain protein.

Gross brain damage grading. Rat pups were decapitated $22 \mathrm{~d}$ after hypoxic exposure. The brains were scored normal, mild, moderate or severe by a blinded observer according to the method of Palmer et al. (20). Normal (0) is no reduction in the size of the right hemisphere, mild (1) is visible reduction in right hemisphere size, moderate (2) is large reduction in hemisphere size from a visible infarct in the right parietal area, and severe (3) is near total destruction of the hemisphere. After removing the cerebellum and brainstem, the brain was divided into two hemispheres and weighed. Results are presented as the percent loss of hemispheric weight of the right side relative to the left [(left - right $) /$ left $\times 100]$. This HI model results in brain damage only on the right side $(4,20)$. There is a high degree of correspondence between the weight deficit of the injured hemisphere and histologically evaluated loss of brain tissue (21).

Microscopic brain damage grading. To verify that the gross changes were a reflection of the expected histopathologic changes, microscopic examination of the tissues after hematoxylin and eosin staining was carried out in 26 rat pups [13 in each group treated with VEGF ( $10 \mathrm{ng}$ ) or vehicle] $3 \mathrm{~d}$ after injury using the previously described method (21). The cerebral cortex, thalamus, and hippocampus were scored by an observer blind to the treatment group of the animal on a range from 0 to 5 according to the method of Cataltepe et al. (22) where " 0 " is normal; " 1 " is $1-5 \%$ of neurons damaged; " 2 " is $6-25 \%$ of neurons damaged; " 3 " is $26-50 \%$ of neurons damaged; "4" is $51-75 \%$ of neurons damaged; and " 5 " is $>75 \%$ of neurons damaged.

Western blot analysis for Akt and ERKs. To determine the effect of VEGF on Akt and ERKs proteins, Akt and ERKs proteins were measured in rat pups undergoing the above neonatal $\mathrm{HI}$ procedure, treated with vehicle or $10 \mathrm{ng}$ of VEGF. At $45 \mathrm{~min}, 6$ and $24 \mathrm{~h}(n=5-7$ in each group) after reoxygenation following HI, the pups were decapitated, brains were removed, and cortexes in both right and left hemispheres were separately dissected and were frozen at $-80^{\circ} \mathrm{C}$. Akt and ERK proteins were assessed as described by Hasegawa et al. (23). Samples with $30 \mu \mathrm{g}$ of protein were denatured and separated electrophoretically and transferred to a PVDF membrane. After incubation in $5 \%(\mathrm{~W} / \mathrm{V})$ nonfat-dried milk in Tris-Buffered saline (TBS) with $0.1 \%$ Tween 20 , the membranes were washed and incubated at $4{ }^{\circ} \mathrm{C}$ overnight with the primary antibodies. Anti-Akt (1:1000), antiphospho-Ser-473 Akt (1:200) and anti-p44/42 MAP kinase (1:500) antibodies were obtained from Cell Signaling Technology (Beverly, MA). Antiphospho ERK-1/2 (1:500) antibody was obtained from Sigma Chemical Co., Aldrich (St. Louis, MO). After washes, the membranes were incubated with 1:3000 diluted secondary antibodies of horseradish peroxidase-conjugated to antirabbit antibody (Amersham Life Science Inc., Heights, IL) or antimouse antibody (Transduction Laboratories, Lexington, KY) for $1 \mathrm{~h}$ at room temperature. The membranes were washed, then incubated in Amersham's Electrochemiluminescence (ECL $\left.{ }^{\mathrm{TM}}\right)$ Western blotting detection reagents and exposed to ECL Hyperfilm (Amersham, IL). Films were scanned using a Logitech Scanman densitometer (Logitech, Inc., Freemont, CA).

Statistical analysis. Categorical variables were analyzed with the $\chi^{2}$ test. Ordinal variables were compared using Mann-Whitney rank sum test. Other variables were expressed as mean \pm SEM and the statistical significance of differences between groups was determined using the $t$ test. Differences were considered significant at $p<0.05$. The size of the groups was chosen to provide an $80 \%$ chance at the 0.05 level of detecting a reduction in percentage (\%) loss of the right brain weight by $1 / 3$ due to HI following i.c.v. VEGF treatment.

\section{RESULTS}

VEGF did not affect temperature, body weight, and mortality. Rectal temperatures were not significantly different between $10 \mathrm{ng}$ of VEGF and vehicle treated pups at any time (group and group by time differences). Body weights of the VEGF treated groups were not significantly different from vehicle treated pups before injury or at $1,4,7,11,14$, or $22 \mathrm{~d}$ after injury. Body weights increased significantly with time in all groups as the pups grew older. Mortality was not significantly different between VEGF (1 of 92 pups, 1.1\%) and vehicle ( 3 of 91 pups, $3.3 \%, p=0.6$ ) treated pups.

Brain water content. Brain water content was measured at $24 \mathrm{~h}$ after HI for rat pups treated with VEGF or vehicle. Brain water content was significantly increased in the right hemisphere in vehicle treated groups relative to the left hemisphere and sham group. Treatment with VEGF (40 ng, but not 5, 10, or $20 \mathrm{ng}$ ) reduced this increase compared with the vehicle $(p<0.05$, Fig. 1).

VEGF significantly increased the levels of brain VEGF at 15 min after injection. Brain VEGF levels were $312.7 \pm 6.2$ $\mathrm{pg} / \mathrm{mg}$ in sham pups. Brain VEGF levels were $555.6 \pm 65.4$ and $370.2 \pm 40.8 \mathrm{pg} / \mathrm{mg}$ at $15 \mathrm{~min}$ after treatment with VEGF

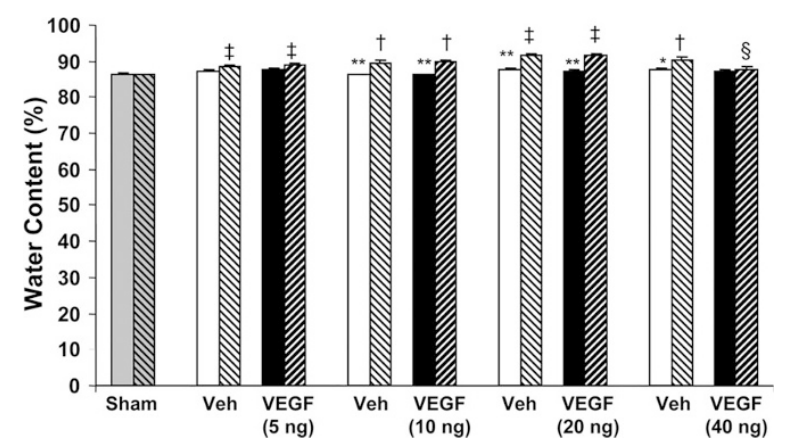

Figure 1. Effect of VEGF on brain water content. Brain water content was measured at $24 \mathrm{~h}$ after HI for groups treated with VEGF ( $\mathbf{\square}$, left hemisphere $(\mathrm{LH}), \boldsymbol{Z}$, right hemisphere $(\mathrm{RH}))$ or vehicle $(\square, \mathrm{LH}, \mathbb{\mathrm { N }}, \mathrm{RH})$ or SHAM ( $\square$, LH, $\mathbb{N}, \mathrm{RH})$. Data are presented as mean \pm SEM $n=5-11$ rat pups for each group. ${ }^{*} p<0.05$, vs. RH, ${ }^{*} p<0.01$, vs. RH, $\uparrow p<0.05$, vs. sham, $\ddagger p<$ 0.01 , vs. sham, and $\S p<0.05$, vs. vehicle. 


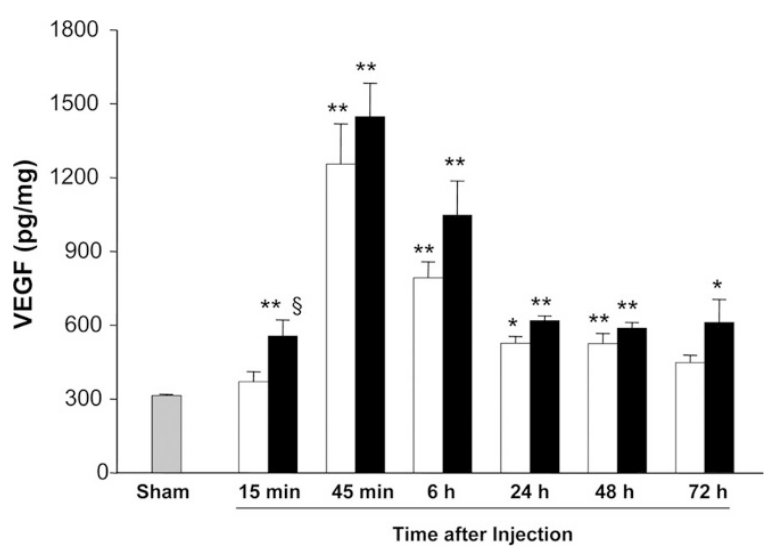

Figure 2. Effect of VEGF on levels of brain VEGF. Brain VEGF levels were measured at 15, $45 \mathrm{~min}, 6,24,48$ and $72 \mathrm{~h}$ after reoxygenation following $\mathrm{HI}$ for groups treated with $10 \mathrm{ng}$ of VEGF $(\square)$ or vehicle $(\square)$. Data are presented as mean \pm SEM, $n=4-19$ rat pups for each group. $* p<0.05$, ** $p<0.01$, vs. sham, $\S p<0.05$, vs. vehicle.

(10 ng) and vehicle, respectively. Treatment with VEGF significantly increased levels of VEGF compared with sham and vehicle groups $(p<0.05$ or $p<0.01$, Fig. 2). At 45 min and $6 \mathrm{~h}$ after treatment, levels of brain VEGF rose 5- to 3-fold in both VEGF and vehicle groups, likely due to HI. Compared with sham, levels of brain VEGF were significantly higher in both VEGF and vehicle groups at 1 and $2 \mathrm{~d}$ as well as in VEGF group at $3 \mathrm{~d}$ after $\mathrm{HI}$.

VEGF (10 and $20 \mathrm{ng}$ ) reduced the decrease in right hemisphere weight. Left hemisphere weight was not affected by the HI, and was not significantly different for vehicle at any dose of VEGF treated pups. The percent reduction in right hemispheric weight caused by HI is shown in Figure 3. VEGF significantly reduced the decrease in right hemisphere weight from $-23.8 \pm 3.8 \%$ in the vehicle treated pups $(n=26)$ to $-11.5 \pm 3.0 \%$ in the pups treated with $10 \mathrm{ng}$ of $\operatorname{VEGF}(n=25$, $p<0.05)$ and from $-20.7 \pm 3.2 \%$ in the vehicle treated pups $(n=29)$ to $-12.1 \pm 2.5 \%$ in the pups treated with 20 ng VEGF

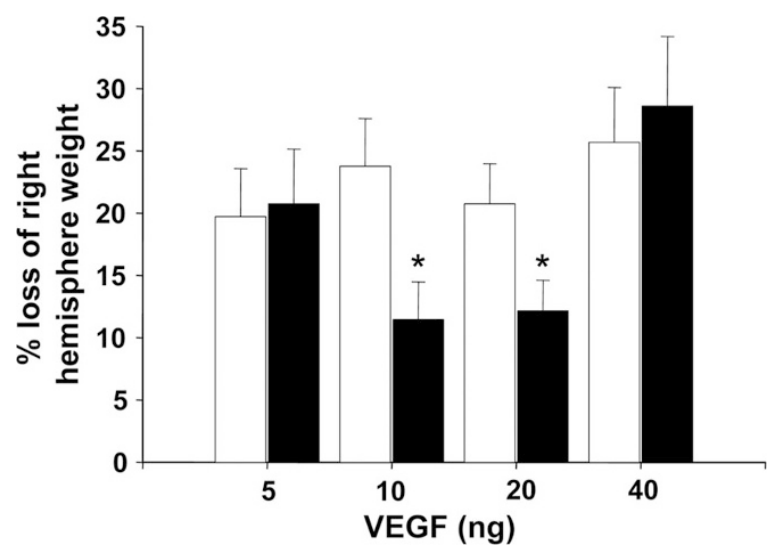

Figure 3. VEGF (10 and $20 \mathrm{ng}$ ) reduced the decrease in right hemisphere weight using the left hemisphere weight as standard. VEGF $(5,10,20$ and 40 ng, $\square$ ) or vehicle $(\square)$ was administered by i.c.v. injection at 5 min after reoxygenation following HI. Brain injury was evaluated $22 \mathrm{~d}$ later. Data are presented as mean \pm SEM, $n=19-33$ rat pups for each group. Treatment with 10 and $20 \mathrm{ng}$ of VEGF significantly decreased the percentage reduction in right hemisphere weight compared with vehicle $(* p<0.05)$. $(n=33, p<0.05)$. Treatment with 5 and 40 ng of VEGF did not affect the reduction in right hemisphere weight.

VEGF (10 and $20 \mathrm{ng}$ ) reduced gross brain injury. The proportion of pup brain scored as normal and damaged (mild, moderate, and severe) is shown in Table 1 . Relative to the vehicle group, the 10 and $20 \mathrm{ng}$ of VEGF treated group had less damage but not treated with the doses of 5 and $40 \mathrm{ng}$ of $\operatorname{VEGF}(p>0.05)$.

VEGF (10 ng) improved the microscopic score in cerebral cortex, thalamus, and hippocampus. The microscopic scores by a blinded observer $3 \mathrm{~d}$ after injury were $0[0,1]$ (VEGF) versus $3[1,4]$ (vehicle, $p<0.02$ ), 0 [0, 1.5] (VEGF) versus $3[0,3.5]$ (vehicle, $p<0.05$ ), and $0[0,2]$ (VEGF) versus 1 [0, 4] (vehicle, $p<0.05$ ) for cortex, thalamus, and hippocampus, respectively (median [25th percentile, 75th percentile], MannWhitney rank sum test) (Fig. 4).

VEGF increased phosphorylation of Akt-Ser-473 and ERK-1/2 at 45 min after reoxygenation following HI. Time course of total Akt, total ERK-1/2 and phosphorylation of Akt-Ser-473 and ERK-1/2 activities were measured in pups treated with $10 \mathrm{ng}$ of VEGF or vehicle and sampled at $45 \mathrm{~min}$, 6 and $24 \mathrm{~h}$ after reoxygenation following HI (Fig. 5). The activities were expressed as a percentage (\%) increase in OD of the right hemisphere relative to the left hemisphere. As shown in Figure $5 A$ and $B$, the Akt-Ser-473 phosphorylation activity was $92.6 \pm 6.1 \%$ in the sham group $(n=5)$ and $110.7 \pm 6.7 \%$ in the vehicle group $(n=6)$ and $132.9 \pm 4.6 \%$ in the VEGF treated group $(n=7)$ at 45 min after reoxygenation. Figure $5 C$ and $D$ show that the phosphorylation of

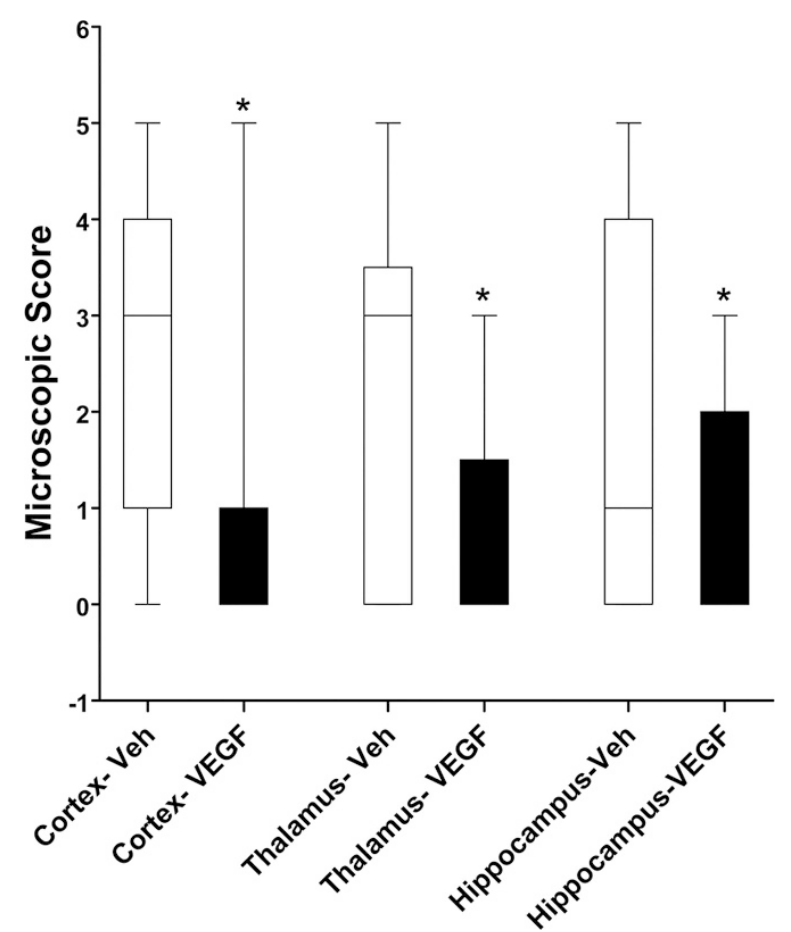

Figure 4. VEGF (10 ng) improved the microscopic score in cerebral cortex, thalamus, and hippocampus. VEGF (10 ng) or vehicle was administered by i.c.v. injection at $5 \mathrm{~min}$ after reoxygenation following HI. Cerebral cortex, thalamus, and hippocampus were scored by a blind observer $3 \mathrm{~d}$ after HI. Data are presented as median [25th percentile, 75 th percentile]. The VEGF treated pups had statistically less damage in cerebral cortex, thalamus and hippocampus $(* p<0.05 \mathrm{vs}$. vehicle, Mann-Whitney rank sum test, $n=13$ for each group). 
A

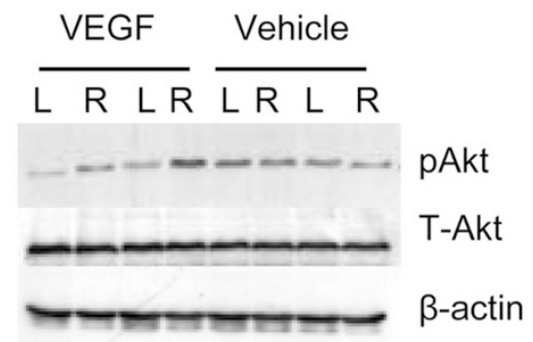

C

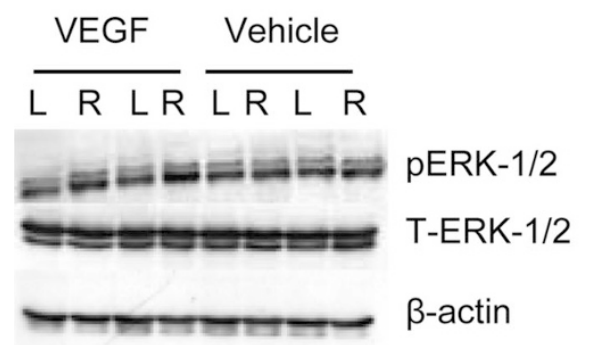

B

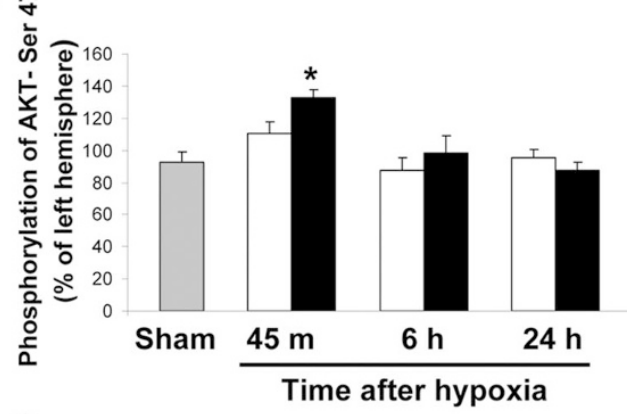

D

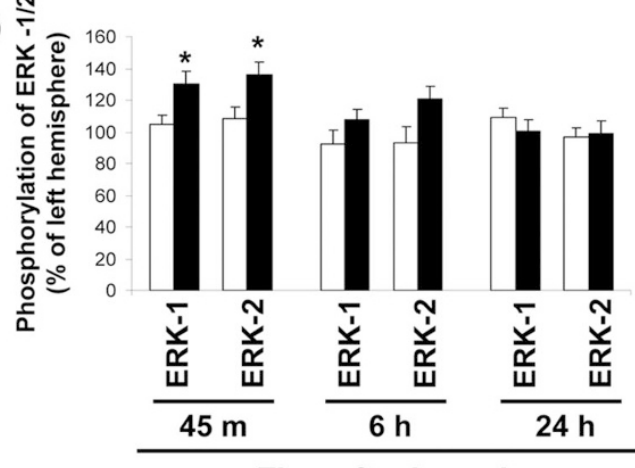

Figure 5. VEGF increased phosphorylation of Akt-Ser-473 and ERK-1/2 at 45 min after reoxygenation following HI. Rat pups treated with $10 \mathrm{ng}$ of VEGF $(\boldsymbol{\square})$ or vehicle $(\square)$. Representative protein bands from Western blots, $A$ : for phosphorylation of Akt-Ser-473 (P-Akt), total Akt (T-Akt) and $\beta$-Actin, $C$ : phosphorylation of ERK1/2 (P-ERK-1/2), total ERK-1/2 (T-ERK$1 / 2$ ) and $\beta$-Actin, sampled at 45 min after reoxygenation. $\mathrm{L}$, left hemisphere; $\mathrm{R}$, right hemisphere. $B$ and $D$ show that treatment with VEGF significantly increased AktSer-473 and ERK-1/2 phosphorylation respectively, at $45 \mathrm{~min}$ after reoxygenation $\left({ }^{*} p<0.05 v s\right.$. vehicle). Treatment with VEGF did not affect Akt-Ser-473 or ERK$1 / 2$ phosphorylation at 6 and $24 \mathrm{~h}$ after reoxygenation following HI. $n=5-7$ rat pups for each group.

ERK-1 (44 kD) and ERK-2 (42 kD) activities were $130.3 \pm$ $8.0 \%$ and $135.7 \pm 8.1 \%$ in the VEGF groups $(n=12)$, and $104.8 \pm 5.8 \%$ and $108.3 \pm 7.3 \%$ in the vehicle group $(n=10)$ at $45 \mathrm{~min}$ after reoxygenation, respectively. Thus, treatment with VEGF significantly increased Akt-Ser-473 and ERK-1/2 phosphorylation at $45 \mathrm{~min}$ after reoxygenation $(p<0.05$ versus vehicle). Treatment with VEGF did not affect Akt-Ser473 (Fig. 5B) and ERK-1/2 phosphorylation (Fig. 5D) at 6 and $24 \mathrm{~h}$ after reoxygenation following $\mathrm{HI}$ as compared with corresponding vehicle treated groups $(p>0.05, n=6-7$ in each). Total Akt and total ERK-1/2 activity did not change between VEGF and vehicle treated groups at any time point $(p>0.05$, data not shown).

\section{DISCUSSION}

The most important finding of the present study is that i.c.v. VEGF given 5 min after reoxygenation following HI reduces brain injury in a neonatal rat model of HI as measured by gross brain damage grading and reduction in right hemisphere weight at $22 \mathrm{~d}$ after injury. The histopathologic changes $3 \mathrm{~d}$ after injury are also consistent with a reduction in delayed brain injury. VEGF treatment also increases Akt and ERKs phosphorylation $45 \mathrm{~min}$ after reoxygenation, which may have played an important role in neuroprotection.

To our knowledge, this is the first study that shows a neuroprotective role of i.c.v. VEGF in neonatal HI. Many studies in adult animals have investigated the effects of exogenous administered VEGF on ischemic brain injury. In an adult rat, temporary middle cerebral artery occlusion (MCAO) model, i.c.v. infusion of VEGF decreased infarct volume and brain edema (9). In an adult mouse MCAO model, a single i.c.v. injection of VEGF ( $8 \mathrm{ng}$ ) given 1 or $3 \mathrm{~h}$ after reperfusion protected the brain against ischemia (11). Interestingly, VEGF has been ineffective or detrimental when administered via the systemic route $(11,24)$ because it causes an immune response and does not cross the blood-brain barrier (BBB) (25). In addition, when given systemically, VEGF likely stimulates luminal receptors that trigger opening of tight junctions or activate matrix metalloproteinases with a resultant increase in BBB permeability and increased neuronal cell death $(11,26,27)$. When given i.c.v., VEGF may be having topical effects in the surrounding brain. A study by Storkebaum et al. (25) showed that after i.c.v. delivery VEGF not only diffused into and accumulated in the adjacent brain parenchyma but remained intact for at least $3 \mathrm{~h}$. Our ELISA data also suggest that VEGF diffuses into the surrounding brain parenchyma after i.c.v. delivery. In summary, doses (10 and $20 \mathrm{ng}$ ), and route (i.c.v.) of VEGF injection in our study are consistent with other reports. It is possible that VEGF may not have achieved therapeutic level at the lower dose of $5 \mathrm{ng}$. VEGF (40 $\mathrm{ng}$ ) reduced cerebral edema due to HI but failed to show late neuroprotection. Reasons for the insufficiency of the higher dose are not entirely clear and demonstrate the need for further investigation. In our model, VEGF was given $5 \mathrm{~min}$ after reoxygenation, which was relatively early compared with most other studies. Abumiya et al. (24) suggest that early VEGF administration after cerebral ischemia aggravates injury but in that study VEGF was given systemically. VEGF does have a wider therapeutic window, at least in an adult mouse MCAO model (11). This may not be the case in the newborn rat as our ELISA data showed an increase in endogenous VEGF within $45 \mathrm{~min}$ of reoxygenation in the newborn. Still, it will be worthwhile to evaluate the therapeutic time window of VEGF in the neonatal HI model in further studies.

VEGF increases vascular permeability, which could result in brain edema and therefore worsen ischemic brain injury 
(28). In our study, we found that VEGF does not cause an increase in brain water content in the brain hemisphere affected by HI. This substantiates a similar finding in adult animals (9). It is possible that when administered i.c.v. it may have a more direct effect on neurons and less effect on endothelial cells. However, the possibility of worsening brain edema after HI brain injury in newborn animals by multiple doses or longer duration of VEGF cannot be completely ruled out.

Survival effects of VEGF on endothelial cells involves activation of the VEGFR-2 receptor and modulation of the phosphatidylinositol 3'- kinase (PI3 K)/Akt signaly pathways (29). Many in vitro and in vivo studies suggest involvement of this pathway in neuroprotection by VEGF $(7,17,18)$. Likely downstream targets for activated Akt include Bcl-2 associated death protein (BAD), caspase 9, members of the Forkhead family of transcription factor (FOXO), CREB and NF- $\kappa$ B $(13,17,18)$. Kilic et al. (18) have shown that VEGF protects axotomized retinal ganglion cells by activating Akt as well as ERKs pathways. ERKs pathways have been implicated previously in neuroprotection by hypoxic preconditioning (16) as well as by other growth factors, such as brain derived neural growth factor (BDNF) and erythropoietin $(16,17)$. In our model, we found that VEGF caused an early and transient increase in Akt and ERKs phosphorylation, which may mediate neuroprotection.

There are limitations to this study. We have not examined whether VEGF remains intact in brain after administration. In addition, the role of ERKs pathways in neuroprotection after $\mathrm{HI}$ is controversial. Our interpretation of increases in ERKs phosphorylation by VEGF as one of the mechanisms of neuroprotection against $\mathrm{HI}$ is speculative. Previous studies showed that cerebral ischemia increased VEGF expression. In our model, we found a much earlier (45 min after reoxygenation) increase in VEGF expression compared with other reports $(6 \mathrm{~h})$. There is thus a question as to whether exogenous VEGF is able to modify neuroprotection in the ischemic brain. Any change in the critical reperfusion period early after HI can have long lasting effects. We believe that our findings of increased VEGF within 15 min, early and transient changes in Akt and ERKs phosphorylation (45 min after reoxygenation) and evidence of later neuroprotection by i.c.v. VEGF treatment corroborates its role in neuroprotection. In addition, there may be a difference in the distribution of endogenously induced and exogenously administered VEGF.

In summary, in a newborn rat model of HI, i.c.v. administration of VEGF had neuroprotective effects possibly associated with activation of Akt and ERKs pathways. These findings show the need for further studies with VEGF to evaluate a therapeutic time window, effects of multiple doses/longer duration of treatment, functional outcome and confirmation of the mechanisms. A major limitation of VEGF treatment is the need for i.c.v. delivery or a similar route to ensure its direct effects on the brain. Strategies to up-regulate VEGF expression very early in response to $\mathrm{HI}$ may provide a better alternative.

Acknowledgment. We thank Dr. Jonathan D. Fratkin, Professor, Department of Pathology, University of Mississippi Medical Center for his expert assistance in histopathology.

\section{REFERENCES}

1. Perlman JM 1997 Intrapartum hypoxic-ischemic cerebral injury and subsequent cerebral palsy: medicolegal issues. Pediatrics 99:851-859

2. Vannucci RC, Perlman JM 1997 Interventions for perinatal hypoxic-ischemic encephalopathy. Pediatrics 100:1004-1014

3. Perlman JM 2006 Intervention strategies for neonatal hypoxic-ischemic cerebral injury. Clin Ther 28:1353-1365

4. Rice JE, Vannucci RC, Brierley JB 1981 The influence of immaturity on hypoxicischemic brain damage in the rat. Ann Neurol 9:131-141

5. Ashwal S, Pearce WJ 2001 Animal models of neonatal stroke. Curr Opin Pediatr 13:506-516

6. Silverman WF, Krum JM, Mani N, Rosenstein JM 1999 Vascular, glial and neuronal effects of vascular endothelial growth factor in mesencephalic explant cultures. Neuroscience 90:1529-1541

7. Jin KL, Mao XO, Greenberg DA 2000 Vascular endothelial growth factor: direct neuroprotective effect in in vitro ischemia. Proc Natl Acad Sci USA 97:10242-10247

8. Jin K, Mao XO, Batteur SP, McEachron E, Leahy A, Greenberg DA 2001 Caspase-3 and the regulation of hypoxic neuronal death by vascular endothelial growth factor. Neuroscience 108:351-358

9. Harrigan MR, Ennis SR, Sullivan SE, Keep RF 2003 Effects of intraventricular infusion of vascular endothelial growth factor on cerebral blood flow, edema, and infarct volume. Acta Neurochir (Wien) 145:49-53

10. Harrigan MR, Ennis SR, Masada T, Keep RF 2002 Intraventricular infusion of vascular endothelial growth factor promotes cerebral angiogenesis with minimal brain edema. Neurosurgery 50:589-598

11. Kaya D, Gursoy-Ozdemir Y, Yemisci M, Tuncer N, Aktan S, Dalkara T 2005 VEGF protects brain against focal ischemia without increasing blood-brain permeability when administered intracerebroventricularly. J Cereb Blood Flow Metab 25:1111-1118

12. Northington FJ, Graham EM, Martin LJ 2005 Apoptosis in perinatal hypoxicischemic brain injury: how important is it and should it be inhibited? Brain Res Brain Res Rev 50:244-257

13. Franke TF, Hornik CP, Segev L, Shostak GA, Sugimoto C 2003 PI3K/Akt and apoptosis: size matters. Oncogene 22:8983-8998

14. Franke TF, Kaplan DR, Cantley LC 1997 PI3K: downstream AKTion blocks apoptosis. Cell 88:435-437

15. Zhao H, Shimohata T, Wang JQ, Sun G, Schaal DW, Sapolsky RM, Steinberg GK 2005 Akt contributes to neuroprotection by hypothermia against cerebral ischemia in rats. J Neurosci 25:9794-9806

16. Jones NM, Bergeron M 2004 Hypoxia-induced ischemic tolerance in neonatal rat brain involves enhanced ERK1/2 signaling. J Neurochem 89:157-167

17. Wick A, Wick W, Waltenberger J, Weller M, Dichgans J, Schulz JB 2002 Neuroprotection by hypoxic preconditioning requires sequential activation of vascular endothelial growth factor receptor and Akt. J Neurosci 22:6401-6407

18. Kilic U, Kilic E, Jarve A, Guo Z, Spudich A, Bieber K, Barzena U, Bassetti CL, Marti HH, Hermann DM 2006 Human vascular endothelial growth factor protects axotomized retinal ganglion cells in vivo by activating ERK-1/2 and Akt pathways. J Neurosci 26:12439-12446

19. Cheng Y, Deshmukh M, D'Costa A, Demaro JA, Gidday JM, Shah A, Sun Y, Jacquin MF, Johnson EM, Holtzman DM 1998 Caspase inhibitor affords neuroprotection with delayed administration in a rat model of neonatal hypoxic-ischemic brain injury. J Clin Invest 101:1992-1999

20. Palmer C, Vannucci RC, Towfighi J 1990 Reduction of perinatal hypoxic-ischemic brain damage with allopurinol. Pediatr Res 27:332-336

21. Feng Y, Fratkins JD, LeBlanc MH 2004 Treatment with tamoxifen reduces hypoxicischemic brain injury in neonatal rats. Eur J Pharmacol 484:65-74

22. Cataltepe O, Vannucci RC, Heitjan DF, Towfighi J 1995 Effect of status epilepticus on hypoxic-ischemic brain damage in the immature rat. Pediatr Res 38:251-257

23. Hasegawa Y, Hamada JI, Morioka M, Yano S, Kawano T, Kai Y, Fukunaga K, Ushio Y 2003 Neuroprotective effect of postischemic administration of sodium orthovanadate in rats with transient middle cerebral artery occlusion. J Cereb Blood Flow Metab 23:1040-1051

24. Abumiya T, Yokota C, Kuge Y, Minematsu K 2005 Aggravation of hemorrhagic transformation by early intraarterial infusion of low-dose vascular endothelial growth factor after transient focal cerebral ischemia in rats. Brain Res 1049:95-103

25. Storkebaum E, Lambrechts D, Dewerchin M, Moreno-Murciano MP, Appelmans S, Oh H, Van Damme P, Rutten B, Man WY, De Mol M, Wyns S, Manka D, Vermeulen K, Van Den Bosch L, Mertens N, Schmitz C, Robberecht W, Conway EM, Collen D, Moons L, Carmeliet P 2005 Treatment of motoneuron degeneration by intracerebroventricular delivery of VEGF in a rat model of ALS. Nat Neurosci 8:85-92

26. Gu Z, Kaul M, Yan B, Kridel SJ, Cui J, Strongin A, Smith JW, Liddington RC, Lipton SA 2002 S-nitrosylation of matrix metalloproteinases: signaling pathway to neuronal cell death. Science 297:1186-1190

27. Zhang Z, Chopp M 2002 Vascular endothelial growth factor and angiopoietins in focal cerebral ischemia. Trends Cardiovasc Med 12:62-66

28. Zhang ZG, Zhang L, Jiang Q, Zhang R, Davies K, Powers C, Bruggen N, Chopp M 2000 VEGF enhances angiogenesis and promotes blood-brain barrier leakage in the ischemic brain. J Clin Invest 106:829-838

29. Gerber HP, McMurtrey A, Kowalski J, Yan M, Keyt BA, Dixit V, Ferrara N 1998 Vascular endothelial growth factor regulates endothelial cell survival through the phosphatidylinositol $3^{\prime}$-kinase/Akt signal transduction pathway. Requirement for Flk-1/KDR activation. J Biol Chem 273:30336-30343 